\title{
ANTIOSTEOPOROTIC ACTIVITY OF ANTHRAQUINONE ISOLATED FROM MORINDA CITRIFOLIA FRUITS IN RATS
}

\author{
ABIN JOY*, CHAITRA N, ASHOK M, HANDRAL M \\ Department of Pharmacology, PES College of Pharmacy, Bengaluru - 560 050, Karnataka, India. Email: abinjoy20@gmail.com
}

Received: 04 June 2016, Revised and Accepted: 08 June 2016

\section{ABSTRACT}

Objectives: This study was designed to investigate the antiosteoporotic activity of isolated anthraquinones from Morinda citrifolia fruit extract in ovariectomy (OVX) induced osteoporotic rats.

Methods: All the rats were divided into 4 groups (n=6 each). Group I (sham control) received vehicle, p.o., Group II OVX control (vehicle, p.o.), Group III was OVX+standard raloxifene (5.4 mg/kg, p.o.), and Group IV was OVX+Physcion (100 mg/kg, p.o.) for 90 days.

Results: The daily oral administration of isolated compound physcion (100 mg/kg) for 12 weeks to the rats prevented OVX-induced osteoporosis. This was examined by serum biomarkers such as alkaline phosphatase, calcium, and tartrate resistant acid phosphatase and showed significant effects $(\mathrm{p}<0.0001)$. The femur bone strength assessed by three-point bending test showed improved bone strength in physcion treated rats, and this was supported by enhanced bone mineral density $(\mathrm{p}<0.05)$. The ash parameters of femur bone studied from physcion treated rats exhibited a significant $(\mathrm{p}<0.0001)$ value of ash weight followed by ash calcium content. Further, femur bone histological examination revealed the protective effect of the compound physcion (100 mg/kg) against OVX-induced bone loss in rats, where it showed mineralization of trabecular spaces, improved bone compactness thereby intact bone architecture.

Conclusion: This study concludes that the isolated anthraquinone physcion had a preventive effect against OVX-induced bone loss in rats.

Keywords: Morinda citrifolia, Physcion, Osteoporosis, Bone mineral density, Ash mineral content.

(C) 2016 The Authors. Published by Innovare Academic Sciences Pvt Ltd. This is an open access article under the CC BY license (http://creativecommons. org/licenses/by/4. 0/) DOI: http://dx.doi.org/10.22159/ajpcr.2016.v9i5.13269

\section{INTRODUCTION}

Osteoporosis is a complex, multifactorial chronic disorder characterized by low bone mass, microarchitectural deterioration of bone tissue leading to enhanced bone fragility resulting in increased fracture risk and also a major cause of morbidity and mortality [1] Although exact numbers are not available, with increasing longevity of the Indian population, it is now being realized that osteoporotic fractures are a major cause of morbidity and mortality in the aging population [2,3]. The occurrence of osteoporosis is increased with the age especially in postmenopausal women because estrogen deficiency is the most common cause of hormone related osteoporosis [4]. The most frequent and important of the related fractures are those of the proximal femur, vertebrae and distal forearm, but fractures commonly occur at many other locations as well. It has been recognized for over a century that hip fractures are a manifestation of osteoporosis in the elderly, and vertebral fractures have been virtually synonymous with postmenopausal osteoporosis [5]. The fracture risk increases exponentially with each decade in age over 50. Residents of nursing homes may be at an even higher risk of fracture. Both men and women lose bone as they age. However, women have accelerated bone loss surrounding menopause due to loss of estrogen. Men have some protection from osteoporosis owing to their large bone mass and size and the absence of menopause [6]. The process of bone remodeling that maintains a healthy skeleton may be considered as a preventive maintenance program, frequently removing older bone and replacing it with new bone. Bone loss occurs when this balance is altered; resulting in greater bone removal than replacement thereby individual trabecular plates of bone are lost, leaving an architecturally weakened structure with significantly reduced mass [7]. Bone resorption markers reflect the level of osteoclastic activity in the bone-remodeling process. Accelerated osteoclastic activity increases bone turnover and is associated with low bone mass in both pre- and post-menopausal women. Elevated levels of resorption markers indicate increased osteoclastic activity and a higher risk for osteoporotic hip fracture, independent of bone mineral density (BMD) [8]. The management of osteoporosis includes preventive therapy where maximizing the bone mass during the formative years and then maintaining bone mass once peak bone mass has been achieved. Among these antiresorptive agents selective estrogen receptor modulators are compounds that bind with estrogen receptors and exhibit estrogen action in some tissues and anti-estrogen action in other tissues [9]. The hormone replacement therapy, initiated at the onset of menopause, has been demonstrated in numerous studies to be capable of improving menopause-related symptoms, while at the same time preventing the loss of bone mass associated with menopause. Nevertheless, due to its side effects, particularly relevant among which is the potential risk of developing breast cancer, the use of this type of medication should not be anticipated as the first-line therapy for osteoporosis [10]. The phytoestrogens, which are found in plants naturally, help to improve few problems that are associated with menopause. They have been investigated to find out their potential benefits in caring age-related, habitual and hormonal conditions. The genus Morinda (Rubiaceae) including the species Morinda citrifolia is made up of around 80 species. M. citrifolia is commonly known as Indian mulberry or noni. The noni plant has been found to contain amino acids, anthraquinones, fatty acids, flavonoids, sterols, and terpenoids. It also serves as a good source of iron, calcium and other minerals which are effective in reducing pain in osteoporosis and in the reduction of swelling in rheumatoid arthritis [11]. The noni seeds are rich in anthraquinones and calcium, keeping in view that this study was conducted to investigate whether Morinda fruit extract may serve as a potential anti-osteoporotic agent in experimentally induced osteoporosis in female rats.

\section{METHODS}

Experimental animals

Female Sprague-Dawley rats (150-200 g) were purchased from Sri Venkateshwara Enterprises, Bengaluru and were maintained in the animal house of PES College of Pharmacy, Bengaluru (CPCSEA Reg. 
no. 600/PO/Ere/S/02/CPCSEA). All the animals were acclimatized for 7 days under standard husbandry conditions, i.e., room temperature of $25 \pm 1^{\circ} \mathrm{C}$; relative humidity $45-55 \%$. The animals had free access to standard rat pellet (Amruth Animal Feeds Pvt. Ltd., Bengaluru, India), with water supplied ad libitum under strict hygienic conditions. The experimental protocols were approved by the Institutional Animal Ethics Committee (PESCP/IAEC/09/2010, Dated: 14/12/2010) and conducted according to CPCSEA guidelines, Government of India.

\section{Acute oral toxicity (AOT)}

The AOT was performed according to the OECD guidelines 423. In brief, the albino female mice of three groups $(n=3)$ were used. Animals of all the groups were fasted for 3-4 hrs before the test and administered 50,300 and $2000 \mathrm{mg} / \mathrm{kg}$ body weight of freshly prepared M. citrifolia fruit extract. Then, the animals were observed immediately and after 30 minutes, periodically during the first $24 \mathrm{hrs}$, with special attention given during the first $4 \mathrm{hrs}$, and daily thereafter, for a total of 14 days. At the end of the $14^{\text {th }}$ day, the animals were sacrificed with anesthetic overdose and dissected for examination of vital organs [12]

\section{Drugs and chemicals}

Raloxifene hydrochloride tablets (Dr. Reddy's, Hyderabad, India), ketamine hydrochloride injection (Neon Laboratories Ltd.), xylazine injection (Indian Immunologicals Ltd., Andhra Pradesh, India), calcium and alkaline phosphatase (ALP) kits (Erba Mannheim, Baddi, India), tartrate resistant acid phosphatase (TRAP) kit (Accurex Biomedical Pvt. Ltd., Mumbai, India), and all other chemicals and reagents were of analytical grade purchased from SD Fine-Chem Ltd., Mumbai, India.

\section{Plant material}

Fresh fruits of $M$. citrifolia were collected from Arvind Trading Company, Bengaluru, in the month of August. The plant was identified and authenticated by Dr. KP Sreenath, taxonomist Department Botany, Bengaluru University, India.

\section{Isolation and identification of the compound}

The dried powdered fruits were refluxed with ethanol (70\%) for 4 days. The same process was repeated for three times, and then the solvent was concentrated under vacuum and filtered. The major constituents were identified by optimization of thin layer chromatography (TLC). The plates were scanned at 254 and $366 \mathrm{~nm}$ using petroleum ether: Ethyl acetate (8:2) solvent system [13]. About $60 \mathrm{~g}$ of extract was chromatographed on silica gel column (60-120 mesh, $600 \mathrm{~g})$ and successively eluted with an increase in their polarity. The fractions were collected, and each fraction was spotted on pre-coated silica gel plate (Merk-60 F254, $0.25 \mathrm{~mm}$ thick) and eluted according to their polarity. Fractions with sufficient yield were selected for further studies. Fraction 11 showed a single spot on the TLC. The structure of the isolated compound was elucidated on the basis of nuclear magnetic resonance (NMR) and mass spectra analysis (Fig. 1) [14].

\section{Induction of osteoporosis by ovariectomy (OVX) \\ Surgical procedure}

After 1 week of acclimatization, the rats to be operated were anesthetized with ketamine ( $80 \mathrm{mg} / \mathrm{kg}$ i.p.) and xylazine $(10 \mathrm{mg} / \mathrm{kg}$ i.p. $)$ In all the groups, operation was made after placing animal on its ventral surface. The place and used surgical equipment were aseptically cleaned. OVX was preceded by a midline dorsal skin incision, $3 \mathrm{~cm}$ long, approximately half way between the middle of the back and the base of the tail. Incisions of the muscles were made bilaterally. After peritoneal cavity was accessed, the ovary was found surrounded by a variable amount of fat. To avoid bleeding, ligation of the blood vessels was done before removal of the ovary. The connection between fallopian tube and the uterine horn was cut, and the ovary was excised. Whereas in sham operated rats, only ovaries were exposed but not excised. Muscle incision was sutured with absorbable catgut, whereas skin wounds were closed bilaterally with three single catgut sutures. Operated animals were given prophylactic amoxicillin ( $25 \mathrm{mg} / \mathrm{kg}$, i.p.) for 4 days

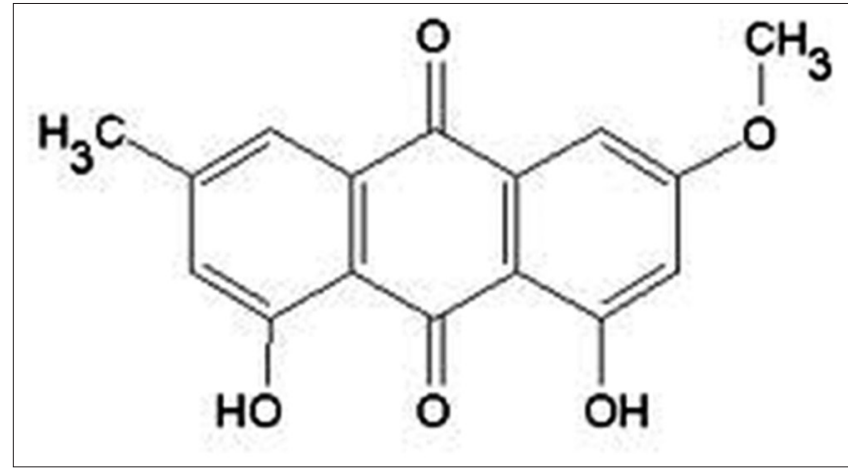

Fig. 1: Structure of physcion

and povidone-iodine solution was applied locally. After surgery, the rats were housed individually in polyurethane cages for a period of 1-week to allow recovery and then re-grouped in their home cages [15].

\section{Experimental design}

Female Sprague-Dawley rats of 6 months old weighing 150-200 g were used in the study. All the operated rats were divided into 5 groups $(n=6)$ and treated for 90 days.

- Group I - Sham operated (vehicle)

- Group II - OVX control (vehicle)

- Group III - OVX + raloxifene $(5.4 \mathrm{mg} / \mathrm{kg}$, p.o.)

- Group IV- Physcion (100 mg/kg, p.o.).

\section{Measurement of length and weight of femur bone}

The femur bones were cleaned from the surrounding tissues then dried, and the length which is defined as the distance between greater trochanter and medial condyle was measured using a digital caliper (Mitutoyo corp., Japan). Then, the bones were kept in an oven and dried at $110^{\circ} \mathrm{C}$ for $8 \mathrm{hrs}$ and the weights of the dried bones were determined using a digital weighing balance (Sartorius AG) [16].

\section{Serum biochemical parameters}

The calcium and ALP were estimated using Erba diagnostic kits, whereas, TRAP was estimated using accurex diagnostic kit.

\section{Three-point bending test}

The isolated femur bones were assessed for their biomechanical strength using three-point bending apparatus. The sample was fixed horizontally between the mounting slots of the apparatus (Zwick/ Roell 2005) against the cylindrical stoppers of $5 \mathrm{~mm}$ diameter where the cylindrical stoppers support the bone at ends which were rigidly fixed to the frame of the apparatus. The load was applied exactly at the center of the bone using a steel wire of $0.5 \mathrm{~mm}$ diameter passing horizontally. The load was varied gradually from $5 \mathrm{~N}$ to maximum breaking point, and the sample values were showed by increments of small steps. Then, the corresponding deflection of the sample was evaluated using a laser displacement sensor through a data acquisition system [17].

\section{Measurement of BMD}

The BMD of rat femur was assessed by fluid displacement method using archimedes' principle [17].

\section{Determination of bone ash weight and mineral content}

Femur bone ash was obtained using muffle furnace $\left(700^{\circ} \mathrm{C}\right.$ for $6 \mathrm{hrs}$ ) and dissolved in $0.1 \mathrm{~mol} / \mathrm{L}$ hydrogen chloride solution. Bone calcium was measured by a ultraviolet-visible spectrophotometer (RMS-BCA 201) [17,18]. 
Table 1: Effect of physcion on change in body weight

\begin{tabular}{|c|c|c|c|c|}
\hline \multirow[t]{2}{*}{ Serial number } & \multirow[t]{2}{*}{ Groups $(n=6)$} & \multicolumn{2}{|c|}{ Change in body weight (g) } & \multirow[t]{2}{*}{ Average increase in body weight $(\mathrm{g})$} \\
\hline & & $1^{\text {st }}$ day & $90^{\text {th }}$ day & \\
\hline 1 & Sham operated & $207.00 \pm 6.68$ & $273.70 \pm 9.41$ & 66.70 \\
\hline 2 & OVX control & $200.70 \pm 7.33$ & $295.00 \pm 4.84^{* * * a}$ & 95.30 \\
\hline 3 & Raloxifene $(5.4 \mathrm{mg} / \mathrm{kg}$ ) & $204.00 \pm 6.21$ & $230.30 \pm 5.35^{* * * b}$ & 26.30 \\
\hline 4 & Physcion (100 mg/kg) & $207.3 \pm 5.43$ & $296.0 \pm 4.58$ & 89.30 \\
\hline
\end{tabular}

Values are in mean \pm SEM $(\mathrm{n}=6)$. One-way ANOVA followed by Tukey-Kramar multiple comparison test ${ }^{* * *} \mathrm{p}<0.0001$. ${ }^{\mathrm{a} N o r m a l}$ versus OVX control, ${ }^{\mathrm{b} O V X}$ control versus treated groups. SEM: Standard error of the mean, OVX: Ovariectomy

\section{Histopathology}

The femur bone was fixed (10\% phosphate formalin buffer) for 24 hrs, decalcified in ethylenediaminetetracetic acid (15\%) for 4 days, dehydrated in alcohol, cleared in xylol, and embedded in paraffin wax $\left(56-58^{\circ} \mathrm{C} \mathrm{mp}\right)$. Sections $(6 \mathrm{~mm})$ were cut with rotary microtome, stained with hematoxylin-eosin, in a jar for 2-20 minutes. The blue staining of the hematoxylin is changed to red by the action of acid. Blue color is regained by washing in alkaline, running tap water for at least 5 minutes. Then, stained in 1\% aqueous eosin for 1-3 minutes excess stain was washed under running tap water for 2-3 minutes and observed under microscope for histopathological changes.

The histopathological images are shown in Fig.2

Statistical analysis

All the values were expressed as a mean \pm standard error of the mean Statistical comparisons were performed by one-way ANOVA followed by Tukey's post-test using GraphPad Prism version 5.0. ${ }^{*} \mathrm{p}<0.05,{ }^{* *} \mathrm{p}<0.01$, ${ }^{* *} \mathrm{p}<0.0001$ was considered as significant compared to disease control.

\section{RESULTS AND DISCUSSION}

\section{AOT}

The AOT study revealed that the extract was found to be safe up to $2000 \mathrm{mg} / \mathrm{kg}$ and further autopsy did not show any toxicity in the vital organs.

\section{Identification of active compound}

The fraction 11 of ethanolic fruit extract of M. citrifolia yielded a yellow colored active compound which was confirmed with its NMR and mass spectra values. These data led to the identification of a well-known compound, physcion, i.e., 1,8-dihydroxy-3methoxy-6methylanthraquinone $\left(\mathrm{C}_{16} \mathrm{H}_{12} \mathrm{O}_{5}\right)$ with a molecular weight of 284 .

\section{Effect of physcion on change in body weight}

The OVX resulted in significant $(\mathrm{p}<0.0001)$ increase in body weight when compared to sham operated rats. Whereas the rats received raloxifene $(5.4 \mathrm{mg} / \mathrm{kg}$ ) exhibited a reduction in body weight $(\mathrm{p}<0.0001)$, but the physcion $(100 \mathrm{mg} / \mathrm{kg})$ did not show any effect on body weight The data are represented in Table 1.

\section{Effect of physcion on femur length and weight}

The treatment with physcion $(100 \mathrm{mg} / \mathrm{kg})$ for 90 days did not show any significant difference in femur length and weight compared to OVX control rats. The data are represented in Table 2.

\section{Effect of physcion on serum biochemical parameters}

The biochemical parameters correlated with the study as characterized by an increase in ALP and TRAP and fall in calcium level. This indicates an increase in the osteoblastic and osteoclastic activity, respectively, thereby showing that bone turnover, which takes place at a faster rate, may account for bone disorder [19]. In this study, groups treated with raloxifene and physcion $(100 \mathrm{mg} / \mathrm{kg})$ showed a suppressed ALP activity, which was highly significant $(\mathrm{p}<0.0001)$ when compared to OVX control rats. The serum calcium was also found to be reduced in OVX control group when compared to sham operated $(\mathrm{p}<0.01)$. Whereas, rats treated with active component physcion $(100 \mathrm{mg} / \mathrm{kg})$
Table 2: Effect of physcion on femur length and weight

\begin{tabular}{llll}
\hline $\begin{array}{l}\text { Serial } \\
\text { number }\end{array}$ & Groups & $\begin{array}{l}\text { Femur } \\
\text { length }(\mathbf{m m})\end{array}$ & $\begin{array}{l}\text { Femur } \\
\text { weight }(\mathbf{g})\end{array}$ \\
\hline 1 & Sham operated & $34.86 \pm 0.257$ & $0.611 \pm 0.005$ \\
2 & OVX control & $35.23 \pm 0.268$ & $0.666 \pm 0.005$ \\
3 & Raloxifene $(5.4 \mathrm{mg} / \mathrm{kg})$ & $35.41 \pm 0.370$ & $0.668 \pm 0.005$ \\
4 & Physcion $(100 \mathrm{mg} / \mathrm{kg})$ & $34.81 \pm 0.249$ & $0.675 \pm 0.005$ \\
\hline \multicolumn{3}{l}{ Values are in mean \pm SEM (n=6). One-way ANOVA followed by Tukey-Kramar }
\end{tabular}

multiple comparison test SEM: Standard error of the mean, OVX: Ovariectomy

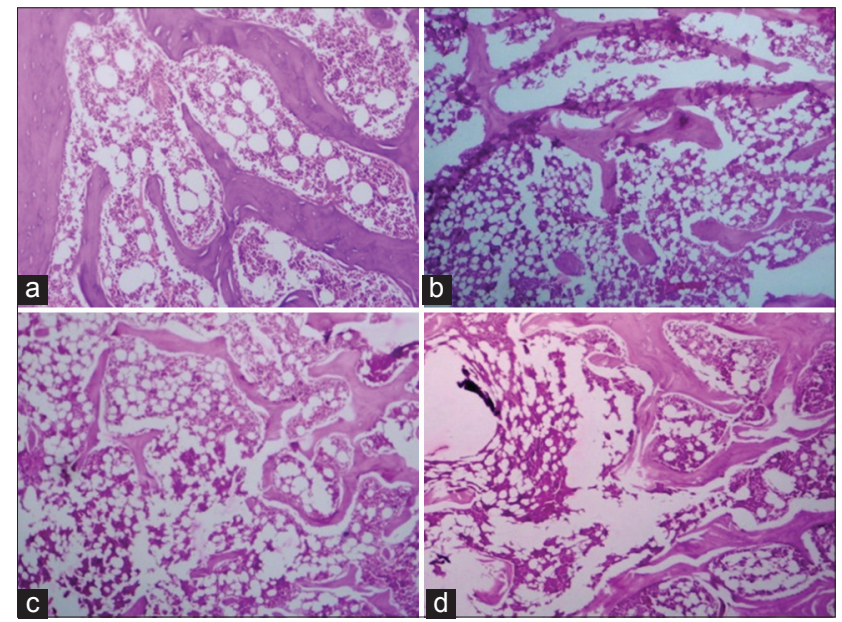

Fig. 2: Histopathology of rat femur bone $(\mathrm{H}$ and $\mathrm{E}, \times 100)$.

(a) Sham operated. (b) Ovariectomy (OVX) control. (c) Raloxifene (5.4 mg/kg). (d) Physcion (100 mg/kg). Slide 1: Sham operated group exhibits normal bone architecture, compact and uniform trabeculae with complete lamella. Slide 2: Section studied form oVX control rat showed loss of bone connectivity. The trabeculae also indicated thinning and loss of lamellae with reduced osteocytes and osteoblasts. Slide 3: The standard raloxifene treated rats showed normal bone architecture with restoration of osteoblasts. Slide 4: Physcion (100 mg/kg) treated group revealed bone with uniform trabeculae thickness and the presence osteoblasts were also evident

exhibited dose-dependent raise in the levels of calcium as compared to OVX control rats $(\mathrm{p}<0.01)$. The TRAP level was significantly increased in OVX control $(\mathrm{p}<0.01)$. But, physcion $(100 \mathrm{mg} / \mathrm{kg})$ supplemented rats showed a significant reduction in level $(p<0.05)$. The effect of physcion on serum biochemical markers are presented in Table 3 .

\section{Effect of physcion on bone mechanical strength and BMD}

BMD has been described as merely a surrogate measure of bone strength. However, microarchitectural properties are a newly emerged marker for the evaluation of the true impact of a treatment on the quality of trabecular bone [20]. The present data (Table 4) indicated that BMD and biomechanical strength were significantly $(\mathrm{p}<0.0001)$ reduced in OVX rats when compared to sham operated group, respectively. In 
Table 3: Effect of physcion on serum biochemical parameters

\begin{tabular}{|c|c|c|c|c|}
\hline Serial number & Groups & ALP (IU/L) & Calcium (mg/dl) & TRAP (IU/L) \\
\hline 1 & Sham operated & $175.6 \pm 17.32$ & $6.643 \pm 0.512$ & $1.65 \pm 0.015$ \\
\hline 2 & OVX control & $66.80 \pm 6.63^{* * * a}$ & $5.162 \pm 0.731^{* * a}$ & $2.53 \pm 0.014^{* * a}$ \\
\hline 3 & Raloxifene $(5.4 \mathrm{mg} / \mathrm{kg}$ ) & $204.5 \pm 0.035^{* * * \mathrm{~b}}$ & $7.193 \pm 0.231^{* * b}$ & $1.62 \pm 0.016^{* * * \mathrm{~b}}$ \\
\hline 4 & Physcion $(100 \mathrm{mg} / \mathrm{kg})$ & $153.20 \pm 5.11^{* * * \mathrm{~b}}$ & $7.37 \pm 0.254^{* * \mathrm{~b}}$ & $2.454 \pm 0.512^{* \mathrm{~b}}$ \\
\hline
\end{tabular}

control versus treated groups. SEM: Standard error of the mean, OVX: Ovariectomy, ALP: Alkaline phosphatase, TRAP: Tartrate resistant acid phosphatase

Table 4: Effect of physcion on bone impact test and bone mineral density

\begin{tabular}{|c|c|c|c|}
\hline Serial number & Groups & Force at break (N) & BMD (wt/ml) \\
\hline 1 & Sham operated & $105.20 \pm 1.92$ & $0.86 \pm 0.013$ \\
\hline 2 & OVX control & $83.57 \pm 4.42^{* * * a}$ & $0.82 \pm 0.015^{* * a}$ \\
\hline 3 & Raloxifene $(5.4 \mathrm{mg} / \mathrm{kg}$ ) & $127.20 \pm 3.78^{* * * \mathrm{~b}}$ & $0.90 \pm 0.005^{* * * \mathrm{~b}}$ \\
\hline 4 & Physcion (100 mg/kg) & $100.35 \pm 0.96^{* * * b}$ & $0.84 \pm 0.014^{* \mathrm{~b}}$ \\
\hline
\end{tabular}

Values are in mean \pm SEM $(\mathrm{n}=6)$. One-way ANOVA followed by Tukey-Kramar multiple comparison test ${ }^{*} \mathrm{p}<0.05,{ }^{* *} \mathrm{p}<0.01,{ }^{* * *} \mathrm{p}<0.0001$. ${ }^{\mathrm{a}}$ Normal versus $0 \mathrm{VX}$ control, ${ }^{\mathrm{b}}$ OVX control versus treated groups. SEM: Standard error of the mean, OVX: Ovariectomy, BMD: Bone mineral density

our study, the physcion $(100 \mathrm{mg} / \mathrm{kg})$ improved both mineral density $(\mathrm{p}<0.01)$ and bone strength $(\mathrm{p}<0.0001)$ to a normal value and which was comparable to the raloxifene-treated rats.

\section{Effect of physcion on bone ash weight and calcium content}

When compared to sham-operated rats, the OVX group exhibited a significant $(\mathrm{p}<0.0001)$ fall in ash weight and calcium content, respectively. Whereas the active component physcion $(100 \mathrm{mg} / \mathrm{kg})$ administered rats showed raise in its ash weight followed by improved calcium content $(\mathrm{p}<0.0001)$. The decreased bone mineral content was observed with ovariectomized rats and further it was evidenced by the reduction of total ash weight and ash calcium content, asserting its role in the prevention of bone loss [21]. The data are represented in Table 5.

Human bone is rich in mineralized organic matrix and various bone cells. Osteoblasts are one among the bone cells that synthesize the organic matrix and regulate the bone formation, whereas osteoclasts cause bone resorption resulting in various problems associated with the skeletal system. The current approach mainly focussed to develop antiosteoporotic drugs which are directed with bone resorption. In most postmenopausal woman, there is a deficiency in the production of ovarian hormone and it has been recommended to improved bone loss. The hormone deficiency can also correlate to the plasma calcium levels which in turn lead to increased bone resorption [22] The ovariectomized rat model is most commonly used in research on postmenopausal osteoporosis. Like humans, rats have a cancellous bone that undergoes remodeling once longitudinal growth has essentially caused. In adult rats, OVX is followed by an increase in bone turnover associated with bone loss and a permanent deficit of bone mass at several skeletal sites including the vertebral bodies, the proximal femur, and the metaphysis of long bones such as distal femur and proximal tibia [23]. The previous studies have been reported on the plant Morinda officinalis and were found to have anthraquinones, which is a potent constituent responsible for estrogen-dependent bone healing activity and joint diseases. Seo et al. reported to have morinda root extracts both suppressor of bone resorption and an enhancer of bone formation in vivo and may have some favorable effects for preventing and treating the osteoporosis induced by sciatic neurectomy. Yan-Bin et al. also studied on M. officinalis root extract for the isolated anthraquinone derivatives and evaluated their antiosteoporotic activity using osteoblast and osteoclastic cells [24].

In our study, the serum biochemical markers were found to possess significant changes in OVX control groups and protective effect was observed with treated groups. Among them, the bone formation marker ALP and resorption marker TRAP were found to have a significant effect. The serum calcium was also reduced with OVX, but the treatment had an effect on maintaining this level.
Table 5: Effect of physcion on bone ash weight and mineral content

\begin{tabular}{|c|c|c|c|}
\hline $\begin{array}{l}\text { Serial } \\
\text { number }\end{array}$ & Groups & Ash weight (g) & $\begin{array}{l}\text { Calcium } \\
\text { (mg/dl) }\end{array}$ \\
\hline 1 & Sham operated & $0.58 \pm 0.01$ & $11.28 \pm 0.41$ \\
\hline 2 & OVX control & $0.36 \pm 0.014^{* * * a}$ & $7.93 \pm 0.22^{* * * a}$ \\
\hline 3 & Raloxifene $(5.4 \mathrm{mg} / \mathrm{kg}$ ) & $0.60 \pm 0.01^{* * * \mathrm{~b}}$ & $11.46 \pm 0.34^{* * * \mathrm{~b}}$ \\
\hline 4 & Physcion (100 mg/kg) & $0.41 \pm 0.01^{* * b}$ & $9.44 \pm 0.45^{* * * b}$ \\
\hline
\end{tabular}

The bone quality is reduced with a higher amount of bone turnover due to the increased osteoclastic activity. Thus, in this study, we carried out BMD, where it reduced in OVX control group due to improved bone loss but the physcion treated group was protected from bone loss by increasing the bone density. The decreased BMD was also supported by three-point bending of the femur bone in OVX rats, whereas the physcion administered group was highly significant and showed enhanced bone strength. The reduced BMD and bone strength may be due to the poor mineral contents in the bone, so we estimated the bone ash weight and ash calcium content. As expected the ash weight and presence of calcium was reduced in OVX control rats, but there was a significant raise in the level of calcium in physcion treated animals. The physcion also had a significant effect on bone histology.

\section{CONCLUSION}

In this study, we have isolated and elucidated an anthraquinone derivative, physcion from ethanolic fruit extract of M. citrifolia, and evaluated for its antiosteoporotic activity in ovariectomized rats. The results of this study indicated that isolated anthraquinone had a significant effect on bone resorption. Although, the inhibitory effect on bone resorption and the association of its action with female hormone is still unclear. Thus, further studies are necessary to elucidate the molecular mechanisms involved in the action, thereby provide a new approach for herbal drug development.

\section{ACKNOWLEDGMENT}

The authors are thankful to vision group of science and technology (VGST), Bengaluru, for funding this project, Dr. J. Saravanan, Principal and Dr. S. Mohan, Director, PES College of Pharmacy, Bengaluru, for supporting this work. 


\section{REFERENCES}

1. Chitme HR, Muchandi IS, Burli SC. Effect of Asparagus racemosus Willd root extract on ovariectomized rats. J Nat Prod 2009;2:16-23.

2. Thokchom S, Chhugani M. A study to find out the prevalence for osteoporosis and osteopenia in pre and post-menopausal women in India: A cross sectional study. IJSR 2015;4(10):270-3.

3. Malhotra N, Mithal A. Osteoporosis in Indians. Indian J Med Res 2008;127(3):263-8.

4. Zeng GF, Zhang ZY, Lu L, Xiao DQ, Xiong CX, Zhao YX, et al. Protective effects of Polygonatum sibiricum polysaccharide on ovariectomy-induced bone loss in rats. $\mathrm{J}$ Ethnopharmacol 2011;136(1):224-9

5. Melton LJ $3^{\text {rd }}$, Chrischilles EA, Cooper C, Lane AW, Riggs BL. Perspective. How many women have osteoporosis? J Bone Miner Res 1992;7(9):1005-10.

6. Dipiro JT, Rotschafer JC, Kolesar JM, Wells BG. Pharmacotherapy, Principles and Practice. New York: McGraw-Hill Companies, Inc.; 2008.

7. NOF. Clinician's Guide to Prevention and Treatment of Osteoporosis. Washington, DC: National Osteoporotic Foundation; 2010.

8. McCormick RK. Osteoporosis: Integrating biomarkers and other diagnostic correlates into the management of bone fragility. Altern Med Rev 2007;12(2):113-45.

9. Olivos L. Selective Estrogen Receptor Modulators (SERMS). Arq Bras Endocrinol Metabol. 2006;50(4):720-34.

10. Rey JR, Cervino EV, Rentero ML, Crespo EC, Alvaro AO, Casillas M. Raloxifene: Mechanism of action, effects on bone tissue, and applicability in clinical traumatology practice. Open Orthop J 2009;3:14-21.

11. Honey J, Neha B, Ranjan B, Birendra S, Thakur A. Scientific basis of noni plant (Morinda citrfolia). Asian J Res Pharm Sci 2012;2(2):45-7.

12. OECD. Guideline for Testing of Chemicals. Acute Oral Toxicity, 420 Adopted. Paris: OECD; 2001.

13. Revathy S, Elumalai S, Benny M, Antony B. Isolation, purification and identification of curcuminoids from Turmeric (Curcuma longa L.) by column chromatography. J Exp Sci 2011;2:21-5.

14. Kumar R, Murugananthan G, Nandakumar K, Talwar S. Isolation of anxiolytic principle from ethanolic root extract of Cardiospermum halicacabum. Phytomedicine 2011;18(2-3):219-23.

15. Khajuria DK, Razdan R, Mahapatra DR. Description of a new method of ovariectomyin female rats. Rev Bras Reumatol 2012;52(3):462-70.

16. Shivakumar K, Mukund H, Rabin P. Evaluation of antiosteoporotic activity of root extract of Rubia cordifolia in ovariectomized rats. Int J Drug Dev Res 2012;4(3):163-72.

17. Srikanta P, Nagarajappa SH, Viswanatha GL, Handral M, Subbanna R, Srinath $\mathrm{R}$, et al. Antiosteoporotic activity of methanolic extract of an Indian herbal formula NR/CAL/06 in ovariectomized rats. JCIM 2011;9(10):1125-32.

18. Hall LE, Shirley RB, Bakalli RI, Aggrey SE, Pesti GM, Edwards HM Jr. Power of two methods for the estimation of bone ash of broilers. Poult Sci 2003;82(3):414-8.

19. Annie S, Prabhu RG, Malini S. Activity of Wedelia calendulacea Less. in post-menopausal osteoporosis. Phytomedicine 2006;13(1-2):43-8.

20. Yogesh HS, Chandrashekhar VM, Katti HR, Ganapaty S, Raghavendra HL, Gowda GK, et al. Anti-osteoporotic activity of aqueous-methanol extract of Berberis aristata in ovariectomized rats. J Ethnopharmacol 2011;134(2):334-8.

21. Liang H, Yu F, Tong Z, Huang Z. Effect of Cistanches herba aqueous extract on bone loss in ovariectomized rat. Int $\mathrm{J}$ Mol Sci 2011;12(8):5060-9.

22. Soni HK, Kandachia JM, Jani DK, Patel GR. Pharmacological investigation of bonton capsule for anti-osteoporotic activity in ovariectomized rat. Int J Pharm Phytopharmacol Res 2013;3(1):52-6.

23. WHO. Guidelines for Preclinical Evaluation and Clinical Trials in Osteoporosis. Geneva: WHO; 1998.

24. Wu YB, Zheng CJ, Qin LP, Sun LN, Han T, Jiao L, et al. Antiosteoporotic activity of anthraquinones from Morinda officinalis on osteoblasts and osteoclasts. Molecules 2009;14(1):573-83. 